\title{
Survival and prognosis of HIV-associated non-Hodgkin-lymophma in the era of combined anti-retroviral therapy, a European multi-cohort study J Bohlius ${ }^{1,2}$
}

\author{
Address: ${ }^{1}$ for the Collaboration of Observational HIV Epidemiological Research Europe (COHERE) Study Group and ${ }^{2}$ Institute of Social and \\ Preventive Medicine, University of Bern, Bern, Switzerland \\ from I I th International Conference on Malignancies in AIDS and Other Acquired Immunodeficiencies (ICMAOI): Basic, Epidemiologic, and Clinical \\ Research \\ Bethesda, MD, USA. 6-7 October 2008 \\ Published: 17 June 2009 \\ Infectious Agents and Cancer 2009, 4(Suppl 2):PIO doi:10.1186/1750-9378-4-S2-PIO
}

This abstract is available from: http://www.infectagentscancer.com/content/4/S2/PIO

(c) 2009 Bohlius; licensee BioMed Central Ltd.

\section{Background}

Since the introduction of combined anti-retroviral therapy (CART), survival of patients with HIV-associated NHL improved dramatically. We examined survival and HIVrelated prognostic factors of patients with HIV-related NHL in the era of cART within the framework of a large European multi-cohort collaborative study.

\section{Methods}

We analyzed the data of HIV-infected adult patients (age $>16$ years) who developed a NHL after January 1, 1998. We included patients who were cART naïve at inclusion and started cART after January 1, 1998. Only patients who started cART at some point during lifetime were included in the present analysis. CART was defined as regimen with at least three antiretroviral drugs. Patients had to have at least one CD4 cell count measurement after January 1, 1998, and before or within 7 days of NHL diagnosis. Survival and prognostic factors were estimated using crude and adjusted Weibull models, with random-effects accounting for heterogeneity between cohorts.

\section{Results}

We observed 67,659 HIV-infected patients. Of 1,176 patients who developed NHL, 847 (72\%) NHL patients from 22 collaborating cohorts across Europe were included in the present analysis. The 329 (28\%) NHL patients who did not meet the inclusion criteria outlined above were excluded. After 1 year, 66 percent (95\% CI $63 \%-70 \%$ ) of patients with NHL other than Primary Brain Lymphoma (PBL) and 54 percent (95\% CI 43\%$65 \%$ ) of patients with PBL were alive. Negative predictive factors for survival were diagnosis of PBL, low CD4 cell count nadir and history of injection drug use.

\section{Conclusion}

In the era of CART two-thirds of patients diagnosed with HIV-related NHL other than PBL survive for longer than 1 year after diagnosis. Survival is poorer in patients diagnosed with PBL.

More advanced immunodeficiency is the dominant prognostic factor for mortality in patients with HIV-related NHL. 